\title{
Local form-motion interactions influence global form perception
}

\author{
J. D. McCarthy • D. Cordeiro • G. P. Caplovitz
}

Published online: 24 April 2012

(C) Psychonomic Society, Inc. 2012

\begin{abstract}
Object motion perception depends on the integration of form and motion information into a unified neural representation. Historically, form and motion perception are thought to be independent processes; however, research has demonstrated that these processes interact in numerous and complex ways. For example, an object's orientation relative to its direction of motion will influence its perceived speed (Georges, Seriès, Frégnac, \& Lorenceau, Vision Research 42:2757-2772, 2002). Here, we investigated whether this local form-motion interaction influences global form processing. In Experiment 1, we replicated the effect of orientation-dependent modulation of speed. In Experiment 2, we investigated whether the perceived speed of local elements could influence the perceived shape of a global object constructed from grouping of those elements. The results indicated that the orientation of local elements indeed influenced the perceived shape of a global object. We propose that inputs from local form-motion processes are one of perhaps many neural mechanisms underlying global form integration.
\end{abstract}

Keywords Motion perception - Form perception . Perceptual grouping $\cdot$ Horizontal connections $\cdot$ Lateral facilitation

The perception of a moving object depends on how our visual system processes both the shape of the object and the direction

Electronic supplementary material The online version of this article (doi:10.3758/s13414-012-0307-y) contains supplementary material, which is available to authorized users.

J. D. McCarthy $\cdot$ D. Cordeiro · G. P. Caplovitz $(\triangle)$

Department of Psychology, Mail Stop 296,

University of Nevada Reno,

Reno, NV 89557, USA

e-mail: gcaplovitz@unr.edu and speed at which it is moving. Understanding how the brain processes and integrates this information into a coherent percept is a fundamental challenge for vision scientists. Here, we investigate the mechanisms underlying form and motion perception, and specifically we examine how local form and motion information influences the overall perceived shape of a moving object.

Historically, form perception and motion perception are thought to be largely independent neural processes. This view was born from an abundance of evidence supporting a categorical boundary between form and motion processing. For example, the magnocellular pathway is more suited to motion detection, whereas the parvocellular pathway is more sensitive to form information (Benardete, Kaplan, \& Knight, 1992; Lee, Pokorny, Smith, \& Kremers, 1994). Additionally, selective damage to visual cortex can lead to deficits in the ability to perceive object motion, while preserving form and shape (i.e., akinetopsia: Vaina, 1989; Zihl, von Cramon, \& Mai, 1983; Zihl, von Cramon, Mai, \& Schmid, 1991), and damage to other areas can lead to somewhat opposite deficits (apperceptive agnosia; Lissauer, 1889). Furthermore, some neurons throughout visual cortex respond identically to motion across their receptive fields, independent of the moving object's shape (Hubel \& Wiesel, 1959, 1962, 1968), and other neurons respond to a given shape characteristic, independent of motion or directional information (Schiller, Finlay, \& Volman, 1976a, b, c).

However, a growing body of evidence is demonstrating that form and motion interact in various and complex ways (Caplovitz \& Tse, 2006, 2007; Georges, Seriès, Frégnac, \& Lorenceau, 2002; Tse, 2006; Tse \& Caplovitz, 2006; Tse \& Hsieh, 2006; Tse \& Logothetis, 2002; Whitney et al., 2003). For example, global form perception can influence the perceived speed of moving objects (Caplovitz \& Tse, 2007; Kohler, Caplovitz, \& Tse, 2009). Specifically, the perceived 
speed of an object made up of perceptually grouped elements is dictated by the overall shape of the grouped object rather than by the speeds of the local elements (Caplovitz \& Tse, 2007; Kohler et al., 2009). In addition, the perceived position of a drifting Gabor pattern can be influenced by the speed and direction of the drift (De Valois \& De Valois, 1988; Shapiro, Lu, Huang, Knight, \& Ennis, 2010; Tse \& Hsieh, 2006). Moreover, Whitney et al. demonstrated that these driftposition interactions lead to a perceived boundary shift for objects formed from drifting Gabors.

Specifically related to the present article, the orientation of an object relative to its direction of motion (Fig. 1a) has been shown to influence the speed at which it appears to move (Georges et al., 2002; Seriès, Georges, Lorenceau, \& Frégnac, 2002). Specifically, at high speeds (peak $\sim 64 \%$ ), an elongated Gaussian blob moving parallel to its orientation will appear to translate faster than if it moves perpendicular to the motion axis. It is hypothesized that this local form-motion interaction arises from the horizontal connections that exist between neighboring neurons with collinearly aligned receptive fields (Field, Hayes, \& Hess, 1993; Georges et al., 2002; Seriès et al., 2002). We note that the effect of orientation on perceived speed is quite different for much slower continuous motion (Castet, Lorenceau, Shiffrar, \& Bonnet, 1993; Krolik, 1934; Metzger, 1936).

In this article, we investigate whether the local formmotion interaction described by Georges et al. (2002) influences global shape perception. Specifically, do the neural processes that dictate how we perceive the shapes of moving objects depend in part on neural mechanisms that mediate local form-motion interactions? To answer this question, we examined the perceived shape of a moving object made up of four small oriented Gaussian blobs, as shown in Fig. 1b.

If local form-motion interactions contribute to global form perception, predictable shape distortions should be observed when objects like those illustrated in Fig. $1 \mathrm{~b}$ are put in motion. This hypothesis is based on the assumption that if the leading and trailing edge elements were in fact to move at different speeds, the shape of the global object would appear distorted. For example, if the object shown at the left of Fig. 1b were to move rightward, the leading edge would be composed of elements oriented parallel to the motion axis (and thus perceived as moving faster), and the trailing edge of elements oriented perpendicular to the motion axis (thus perceived as moving slower). If the perceived speeds of local elements contribute to global form analysis, the overall perceived shape should be stretched relative to the veridical shape. Similarly, the converse effect (compression) should occur if the leading elements are perpendicular to the motion axis and the trailing edge is parallel to the motion axis. If this is the case, we can conclude that the mechanisms underlying local form-motion interactions precede and influence the construction of global form. However, if global form analyses were independent of

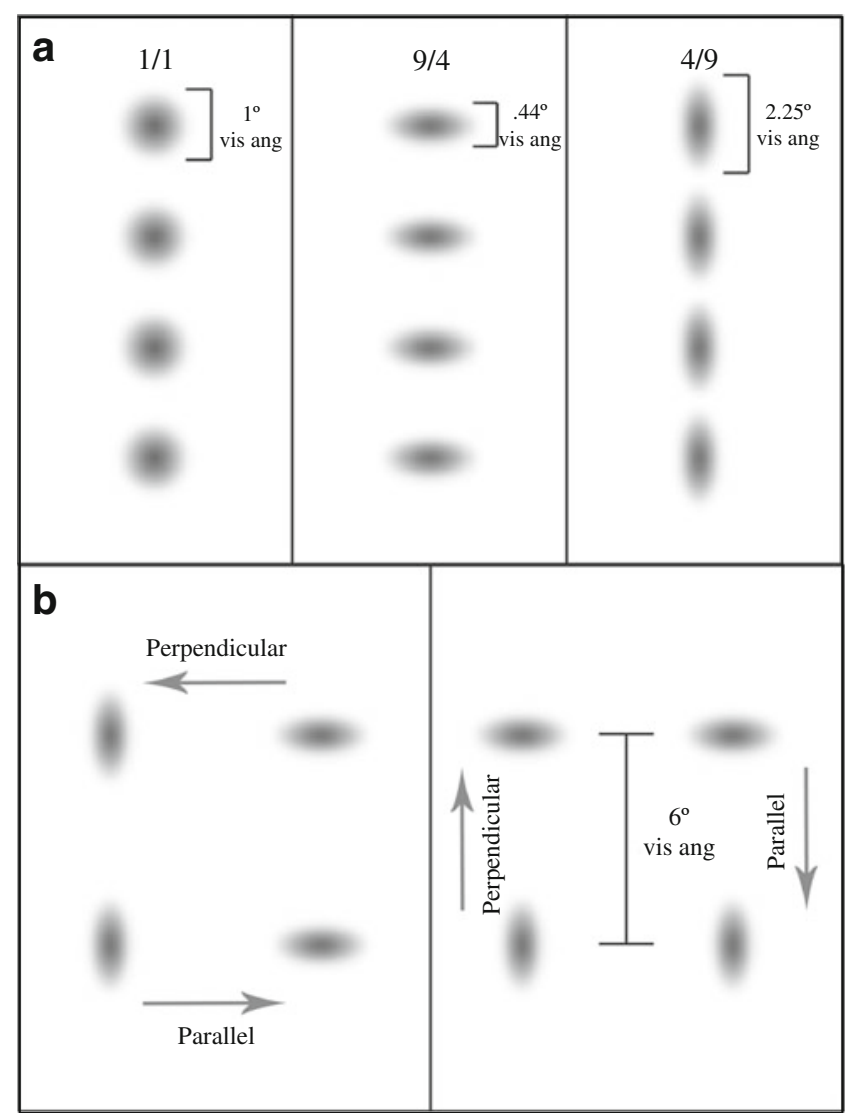

Fig. 1 a Stimuli used in Experiment 1. The left column shows the Gaussian blobs used as the reference stimulus in each condition. The middle and right columns show, respectively, the horizontally and vertically elongated Gaussian blobs used as the test stimuli. b Stimuli used in Experiment 2 . The horizontally and vertically elongated Gaussian blobs from Experiment 1 were arranged to create a global object. This figure shows the condition in which the aspect ratios formed a square (i.e., $6^{\circ} \times 6^{\circ}$ ). When the leading edge of the stimulus was consistent with the direction of motion, the configuration was said to be parallel. When the leading edge of the stimulus was inconsistent with the direction of motion, the configuration was said to be perpendicular. The left of panel $1 \mathrm{~B}$ illustrates the parallel and perpendicular leading-edge conditions when the stimulus traversed a horizontal motion axis. The right of panel 1B illustrates the parallel and perpendicular leading-edge conditions when the stimulus traversed a vertical motion axis

local form-motion interactions, no shape distortions would be expected. In the following two experiments, we first quantify the effect of local orientation information on perceived speed and then, by investigating the perceived shapes of stimuli like those shown in Fig. 1b, demonstrate that these local speed differences lead to global shape distortions.

\section{General method}

Participants

Seven observers participated in the first experiment, and six observers participated in the second experiment. The participants 
provided informed consent in accordance with the Institutional Review Board of the University of Nevada, Reno. They reported normal or corrected-to-normal vision, were naive to the aims and specifics of the experiments, and received course credit for participating.

\section{Apparatus and display}

All stimuli were displayed on an $85-\mathrm{Hz}$ CRT monitor (Dell Trinitron P991, 19 in., 1,024 $\times$ 768) and were generated and presented using the Psychophysics Toolbox (Brainard, 1997) for MATLAB (MathWorks Inc., Natick, MA). Each participant placed his or her head in a chinrest and viewed the stimuli binocularly from a distance of $57 \mathrm{~cm}$

\section{Experiment 1}

The goal of the first experiment was to replicate the effect of orientation-dependent speed modulation demonstrated by Georges et al. (2002) - specifically, their finding that the orientation of a Gaussian blob relative to the motion axis influences its perceived speed.

\section{Method}

Stimuli and procedure Using the method of constant stimuli, participants made two-interval forced choice (2IFC) judgments of the perceived speed of apparentmotion stimuli. The stimulus in each interval was a Gaussian blob (mean luminance $=59 \mathrm{~cd} / \mathrm{m}^{2}$ ) that was presented at spatially sequential locations upward or downward along the vertical axis and against a black background (mean luminance $=.05 \mathrm{~cd} / \mathrm{m}^{2}$ ) (Fig. 1a). The stimulus was shown at each position for two frame refreshes $(\sim 23.5 \mathrm{~ms})$ with a 0 - $\mathrm{ms}$ interstimulus interval (ISI). Each trial consisted of two intervals: a reference and a test, randomly ordered (500-ms ISI). The reference interval consisted of a circular Gaussian blob (standard deviation $=1^{\circ}$ ) with an apparent motion speed of $64^{\circ} / \mathrm{s}$. The test interval consisted of a Gaussian blob that was circular (standard deviation $=1^{\circ}$ ) or was elongated either vertically (aspect ratio $=4 / 9$ ) or horizontally $($ aspect ratio $=$ $9 / 4$ ). Speed was controlled by varying the spatial separation between successive positions. The speed of the test interval was selected from the following list: $16 \%$ s, $32^{\circ} / \mathrm{s}, 48^{\circ} / \mathrm{s}, 64 \%$, $80 \%$, $96 \%$, or $112^{\circ} / \mathrm{s}$, corresponding to spatial separations of $0.38^{\circ}, 0.75^{\circ}, 1.13^{\circ}, 1.5^{\circ}, 1.88^{\circ}, 2.25^{\circ}$, or $2.63^{\circ}$, respectively. The number of positions traversed in an interval varied randomly from three to five $(70.5,94$, or $117.5 \mathrm{~ms})$ in order to prevent judgments based on the duration or length of the motion sequence. On a given trial, the duration and direction of the reference interval was not necessarily the same as that of the test interval. Each trial began with a central fixation point (colored green; $0.35^{\circ}$ ) for $500 \mathrm{~ms}$, after which the two intervals were presented. Participants were instructed to maintain fixation and to indicate by pressing one of two buttons the interval in which motion was perceived to be faster.

In total, 21 different conditions (three orientations and seven speeds) were presented. Each participant completed 420 pseudorandomly presented trials (20 trials of each condition). The participants were given a break after every 100 trials, and they completed 30 practice trials prior to the experiment that were not included in the analyses.

Results

The number of times that the test stimulus was perceived to move faster than the reference was recorded for each condition. Thus, seven values were calculated for each of the three test stimuli. Weibull functions were fit to the corresponding data, with the ceiling of the fitting function set to the first data point for each participant: $f(x)=\operatorname{data}(1)-e^{-(x / a)^{b}}$. The fits of the data for each participant were quite good (mean $R^{2}=$ $.934, S E M=.013$ ), and no significant differences were observed between the goodnesses of fit for the different conditions [repeated measures ANOVA: $F(2,12)=$ 0.434 , n.s.]. The point of subjective equality (PSE; i.e., the speed at which the test stimulus must move in order to be perceived as moving at the same speed as the reference) was computed by interpolating the $50 \%$ point for each curve. The raw data averaged across subjects are shown in Fig. 2, along with curves fit to the mean data. The inset of Fig. 2 illustrates the PSEs for each condition, averaged across subjects.

A repeated measures ANOVA on the PSEs revealed a significant main effect of shape $[F(2,12)=15.36, p<.001$, $\left.\eta^{2}=.72\right]$. Consistent with Georges et al. (2002), a Gaussian blob oriented parallel to the motion axis was perceived to move faster than one oriented perpendicular to the motion [mean difference $\sim 15^{\circ} / \mathrm{s} ; t(6)=5.197, p<.01$ ] or than one containing no orientation information [mean difference $\sim 16 \%$ s; $t(6)=4.56, p<.01]$. This effect is comparable to that derived by Georges et al. using similar stimuli. However, unlike the results of Georges et al., no difference was observed between the perpendicular and circular Gaussians $[t(6)=0.58$, n.s.]. It is unclear why this discrepancy exists, and it could perhaps depend on subtle differences in the experimental designs. Importantly, having demonstrated that parallel orientations can influence perceived speed relative to both circular and perpendicular stimuli, we next investigated whether this effect could influence the perceived shape of a moving object composed of such elements. 
Fig. 2 Results of Experiment 1. The symbols indicate the percentages of trials in which the participants judged the reference to be faster than the test sequences for each condition (averaged across all participants). The solid curves illustrate the fits of the averaged data. The inset of the figure illustrates the points of subjective equality (PSEs) for the three curves. Asterisks indicate significance at the $p<.01$ level, and error bars represent \pm 1 SEM

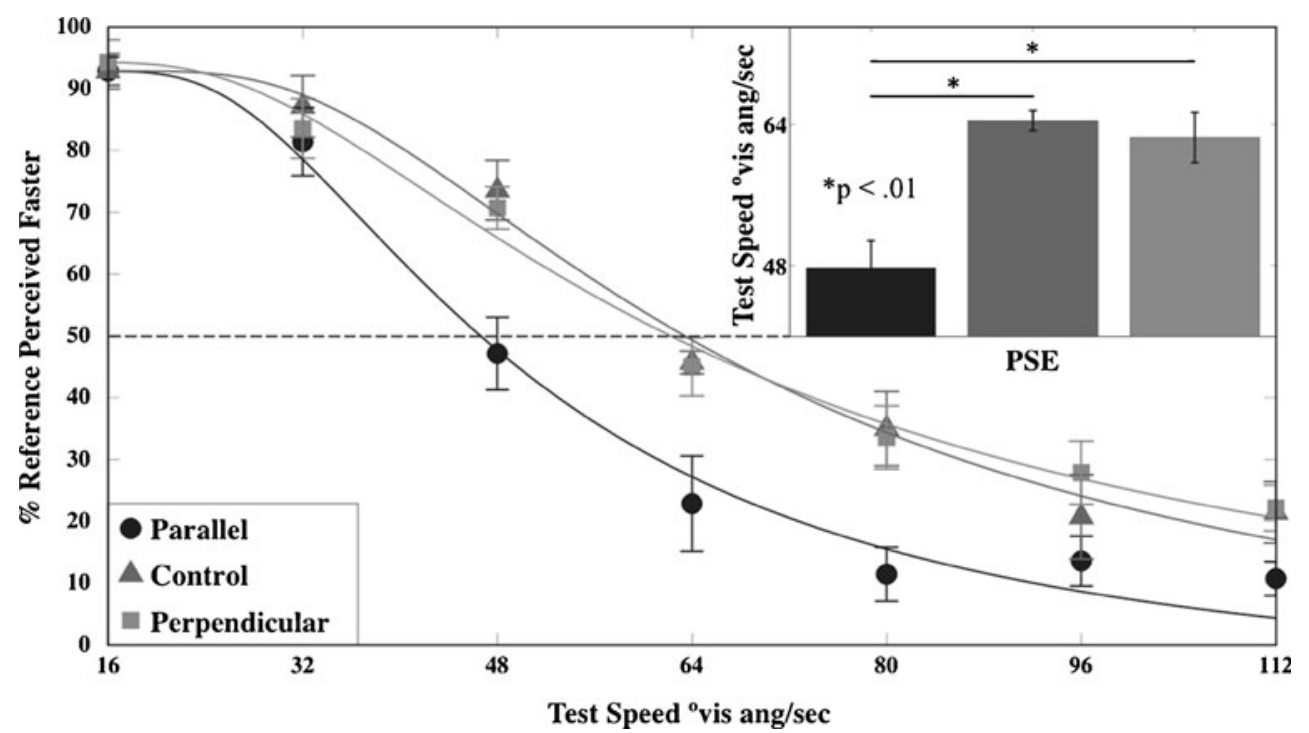

\section{Experiment 2}

Here, we tested whether the aspect ratio of a rectangular object would appear distorted as a function of the orientations, relative to the motion axis, of the corner elements comprising the leading and trailing edges. If the effects observed in Experiment 1 contribute to global shape processing, rectangles in which the leading-edge elements appear to move faster than those in the trailing edge should appear elongated.

\section{Method}

Stimuli and procedure Four elongated Gaussian blobs, arranged to form the corners of a rectangle, moved across the screen. The participants judged the orientation (either horizontal or vertical) of the rectangular array in a single interval. On each trial, two of the blobs were elongated perpendicular to the path of motion, and two were oriented parallel to the path of motion. Here we tested two distinct categories of rectangles (Fig. 1b): one in which the two leading-edge blobs were oriented parallel (with the trailing edge oriented perpendicular), and a second with the two leading-edge blobs oriented perpendicular (with the trailing edge oriented parallel) to the motion axis.

The size of each rectangular array was chosen from the following list on each trial: $3^{\circ} \times 6^{\circ}, 4.5^{\circ} \times 6^{\circ}, 5.4^{\circ} \times 6^{\circ}, 6^{\circ} \times 6^{\circ}$, $6.6^{\circ} \times 6^{\circ}, 7.5^{\circ} \times 6^{\circ}, 9^{\circ} \times 6^{\circ}$. Each array traversed either a horizontal or a vertical apparent motion trajectory at $64 \%$. The apparent motion sequence was composed of six (23.5-ms) steps (each separated by $1.5^{\circ}$ ), for a total stimulus duration of $141 \mathrm{~ms}$. Importantly, the aspect ratios given above are relative to the direction of motion. Thus, if the trajectory was horizontal, the vertical distance between the blobs was always $6^{\circ}$, and if the trajectory was vertical, the horizontal distance between the blobs was $6^{\circ}$. The direction of motion (left, right, up, or down) was randomized on every trial. This uncertainty was used to prevent judgments based on local strategies for performing the task (e.g., judging the distance between the topmost two elements). In total, there were 14 trial types: the two leading-edge configurations in seven aspect ratios. A total of 20 trials of each condition were pseudorandomly presented, resulting in 280 total trials.

\section{Results}

The number of times that participants perceived the global object to be compressed relative to the axis of motion was calculated (i.e., if the stimulus moved horizontally, was it perceived to be oriented vertically?). The data were fit using the same procedures used in Experiment 1 (mean $R^{2}=.925$, $S E M=.027)$, and no significant difference in goodness of fit was observed between the conditions, according to a pairedsamples $t$ test: $t(5)=1.71$, n.s. Figure 3 illustrates the raw data averaged across subjects and the corresponding PSEs (50 \% chance $=$ when participants indicated a perceived square) for the two conditions, as well as data collected in a supplementary control experiment using unoriented Gabors (see the supplementary materials for the experimental details and statistical analyses). When the leading edge was oriented parallel to the motion axis, the stimulus appeared stretched $\sim 0.65^{\circ}$ of visual angle along the direction of motion: One-sample $t$ tests revealed that the PSEs for the parallel leading-edge condition were significantly different than the value for a square $[t(5)=$ $-2.9, p<.05]$. However, no such effect was observed for the perpendicular leading-edge condition $[t(5)=0.2$, n.s.]. A paired-samples $t$ test between the parallel and perpendicular conditions revealed a significant $[t(5)=-3.147, p<.026]$ effect of leading/trailing-edge orientation on perceived shape. Specifically, the shape of a global object with leading-edge 


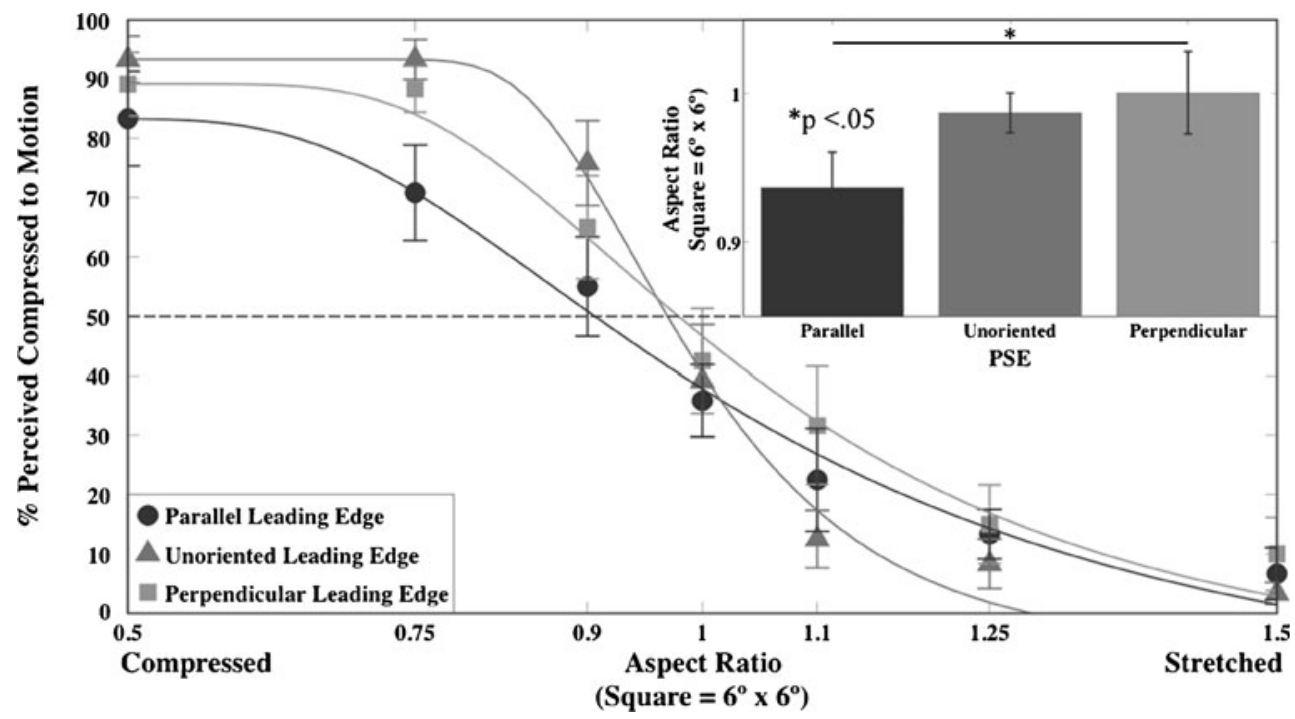

Fig. 3 Results of Experiment 2 and of the first supplementary control experiment. The symbols indicate the percentages of trials in which the participants judged the orientations of the stimuli to be compressed, relative to the motion axis, for the two conditions (averaged across all participants). The solid curves indicate the fits of the averaged data. The data for Experiment 2 are shown as black circles and light gray squares, and the data for the supplementary control experiment are

elements oriented parallel to the motion axis appeared stretched relative to when the leading edge was composed of perpendicular elements. However, these results revealed an unexpected asymmetry, in that the parallel trailing-edge elements did not lead to shape compression.

There are at least two hypotheses as to why this asymmetry may exist. It could be that the effects of local orientation are mediated in part by attentionally driven prediction effects that are biased toward the leading edge (Roach, McGraw, \& Johnston, 2011). To investigate this possibility, we repeated Experiment 2 using circular rather than oriented Gaussian blobs. Here, the hypothesis predicted a distortion in the shape of the stimulus, as the attention-predictive effects were not thought to be exclusively orientation-dependent. However, the results of the circular-blob experiment revealed no significant changes in perceived shape (see the "unoriented leading edge" data in Fig. 3).

An alternative hypothesis is born out of the positions of the trailing-edge elements in the apparent motion sequences. At $64^{\circ} / \mathrm{s}$, each element "jumps" $1.5^{\circ}$ from one step to the next in the sequence. As such, starting with the second step, the trailing-edge elements in the $6^{\circ} \times 6^{\circ}$ square configuration will occupy locations previously occupied by the leading-edge elements. Because the leading- and trailing-edge elements always have orthogonal orientations, this "imprinting" is likely to interfere with whatever collinear facilitatory effects may arise from the trailing edge, thereby limiting the local-orientation effects to the un-interfered-with leading edge. shown as dark gray triangles. The inset of the figure shows the points of subjective equality (PSEs) for the parallel and perpendicular leading-edge conditions (when participants reported that the object was horizontally or vertically oriented at a $50 \%$ chance level). Asterisks indicate significance at the $p<.05$ level, and error bars represent \pm 1 SEM

The primary observation that we present here, though, is that the orientations of individual elements (at least along the leading edge) can influence the perceived shape of an object that they comprise. For completeness' sake, we wanted to rule out the possibility that this effect arises due to the inability of observers to accurately perceive the "aspect ratio" of a rectangle formed out of these oriented elements, rather than to the motion of the elements. We therefore repeated Experiment 2 (including the circular elements) using stationary stimuli (i.e., the elements on a given trial never moved). Unlike the case in which they were moving, no significant differences in perceived shape were observed when the stimuli were stationary (Supplementary Fig. 1).

Taken together, these data support the hypothesis that mechanisms underlying local form-motion interactions precede and contribute to constructing global form. Furthermore, they substantially contribute to the overall perceived shape of a moving object.

\section{Discussion}

The purpose of this research was to investigate whether local form-motion interactions influence global shape perception. These experiments were based on previous research that demonstrated that an object's orientation relative to the motion axis influences its perceived speed (Castet et al., 1993; Georges et al., 2002). Here, we demonstrated that this local form-motion interaction contributes to global shape 
perception. This illustrates that not only do form and motion interact with each other at a local level of processing, but these interactions also contribute to the higher-level perceptual processes that construct global form. These findings are consistent with previous research (Shipley \& Kellman, 1994, 1997) demonstrating that local motion signal extraction precedes global motion perception and boundary formation.

A fundamental question that arises from the present results is the degree to which the perceived speeds of the elements contribute to global shape perception. The data suggest that the global shape is not determined solely by the perceived speeds of the elements: If the leading- and trailing-edge elements were in fact moving with speeds that differed by $15^{\circ} / \mathrm{s}$ (as in Exp. 1), at the end of each trial an object that started as a $6^{\circ} \times 6^{\circ}$ square would have stretched by $\sim 2.12^{\circ}$, nearly three times the observed distortion.

This underestimation can perhaps be accounted for on the basis of the Seriès et al. (2002) model for long-range horizontal connections. The critical characteristic of the model that differentiates it from the description above is that the latency advances accounting for the shifts in perceived speed are expected to saturate over time and distance. Using the model parameters of Seriès et al. (see their Fig. 7B), and assuming a saturated latency advance of $25 \mathrm{~ms}$ for the 6th step in our stimulus array, the model predicts no less than a $\sim 1.6^{\circ}$ distortion $\left(25 \mathrm{~ms} \times 64^{\circ} / \mathrm{s}\right)$, again much greater than the distortion observed in Experiment 2. However, a number of factors, such as contrast and location in the visual field, can influence the strength and speed of the long-range horizontal connections that presumably underlie the results of Experiment 1 . As such, it is difficult to fully interpret the results of Experiment 2 in the context of the Seriès et al. model. Future research will be necessary to fully investigate whether the neural mechanisms underlying effects reported by Georges et al. (2002) are also responsible for the results obtained in Experiment 2.

What is more likely is that global form perception relies on multiple sources of information, of which local speed is but one, albeit a strongly contributing source (Bulakowski, Bressler, \& Whitney, 2007; Whitney, 2002; Whitney et al., 2003). Other sources of information may arise, for example, from an analysis of the retinotopic spacing of activations across the visual field (Inoue, 1909/2000; Murray, Boyaci, $\&$ Kersten, 2006). In the stimuli used here, all four elements that make up the global shape are simultaneously present in the display. As such, neural mechanisms that process the relative positions of the elements independently of their apparent motion may offset the mechanisms underlying the distortions that we report here. The relatively small distortion reported here suggests that these sources of positional information get integrated with local velocity information (De Valois \& De Valois, 1988; Shapiro et al., 2010; Tse \& Hsieh, 2006; Whitney, 2002; Whitney et al., 2003). It has been demonstrated that V4 is a site for the integration of many types of visual information (Desimone \& Schein, 1987). V4 receives input primarily from $\mathrm{V} 2$ projections containing information about visual features such as size and orientation (Deyoe \& van Essen, 1985; Hubel \& Livingstone, 1985; Shipp \& Zeki, 1985). This V1 $\rightarrow$ V2 $\rightarrow$ V4 pathway plays a critical role in object recognition (Desimone \& Schein, 1987). Additionally, V4 receives input from area V3 (Ungerleider, Desimone, \& Moran, 1986; Ungerleider, Gattas, Sousa, \& Mishkin, 1983), which contains motion-sensitive neurons (Burkhalter, Felleman, Newsome, \& van Essen, 1986). The V1-V4 pathway, along with areas MT, MST, and VIP, plays a major role in the ability to judge spatial relationships (Desimone \& Schein, 1987; Ungerleider \& Desimone, 1986).

The local-orientation-specific interaction of form and motion may also be explained in part by biased feedforward connections to area MT. Long-range horizontal connections in V1 facilitate processing for similarly tuned neurons that respond to an object that is oriented parallel to the motion axis (Field et al., 1993; Georges et al., 2002; Seriès et al., 2002). Neurons in MT that encode perceived speed would subsequently be influenced by the biases in the inputs that they receive from V1 (Georges et al., 2002; Seriès et al., 2002). It is possible that in the processing of global form and motion, these biased motion signals arising in V1 and MT become integrated with other, multiple sources of visual information in area V4 concerning the spatial relationships that comprise a global form (Berzhanskaya, Grossberg, \& Mingolla, 2007; Brincat \& Connor, 2006; Ditchfield, McKendrick, \& Badcock, 2006; Francis \& Grossberg, 1995; Van Essen \& Gallant, 1994). Thus, the initial misperception of speed may contribute an inaccurate representation of the spatial relationships that form the global object at higher levels of visual processing.

From the findings of this study, we concluded that discrepant and illusory local-motion signals can lead to a distorted percept of global form. Importantly, the illusory local-motion signals can arise because of a specific interaction between the form (or orientation) and motion of the local element. These results add to a growing body of evidence that form information and motion information interact in various and complex ways across multiple levels of visual processing.

Author Note We thank Morgan Manson for her contributions to this project. We also thank Michael Morgan and three anonymous reviewers for their helpful comments.

\section{References}

Benardete, E. A., Kaplan, E., \& Knight, B. W. (1992). Contrast gain control in the primate retina: $\mathrm{P}$ cells are not $\mathrm{X}$-like, some $\mathrm{M}$ cells are. Visual Neuroscience, 8, 483-486.

Berzhanskaya, J., Grossberg, S., \& Mingolla, E. (2007). Laminar cortical dynamics of visual form and motion interactions during 
coherent object motion perception. Spatial Vision, 20, 337-395. doi:10.1163/156856807780919000

Brainard, D. H. (1997). The psychophysics toolbox. Spatial Vision, 10, 433-436. doi:10.1163/156856897X00357

Brincat, S. L., \& Connor, C. E. (2006). Dynamic shape synthesis in posterior inferotemporal cortex. Neuron, 49, 17-24.

Bulakowski, P. F., Bressler, D. W., \& Whitney, D. (2007). Shared attentional resources for global and local motion processing. Journal of Vision, 7(10), 1-10.

Burkhalter, A., Felleman, D. J., Newsome, W. T., \& van Essen, D. C. (1986). Anatomical and physiological asymmetries related to visual area V3 and VP in macaque extrastriate cortex. Vision Research, 26, 63-80.

Caplovitz, G. P., \& Tse, P. U. (2006). The bar-cross-ellipse illusion: Alternating percepts of rigid and non-rigid motion based on contour ownership and trackable feature assignment. Perception, 35, 993-997.

Caplovitz, G. P., \& Tse, P. U. (2007). Rotating dotted ellipses: Motion perception driven by grouped figural rather than local dot motion signals. Vision Research, 47, 1979-1991.

Castet, E., Lorenceau, J., Shiffrar, M., \& Bonnet, C. (1993). Perceived speed of moving lines depends on orientation, length, speed and luminance. Vision Research, 33, 1921-1936.

Desimone, R., \& Schein, S. J. (1987). Visual properties of neurons in area V4 of the macaque: Sensitivity to stimulus form. Journal of Neurophysiology, 57, 835-867.

De Valois, R. L., \& De Valois, K. K. (1988). Spatial vision. New York: Oxford University Press.

Deyoe, E. A., \& van Essen, D. C. (1985). Segregation of efferent connections and receptive field properties in visual area V2 of the macaque. Nature, 317, 58-61.

Ditchfield, J. A., McKendrick, A. M., \& Badcock, A. R. (2006). Processing of global form and motion in migraineurs. Vision Research, 46, 141-148.

Field, D. J., Hayes, A., \& Hess, R. F. (1993). Contour information in the human visual system: Evidence for a local "association field. Vision Research, 33, 173-193.

Francis, G., \& Grossberg, S. (1995). Cortical dynamics of form and motion integration: Persistence, apparent motion and illusory contours. Vision Research, 36, 149-173.

Georges, S., Seriès, P., Frégnac, Y., \& Lorenceau, J. (2002). Orientation dependent modulation of apparent speed: Psychophysical evidence. Vision Research, 42, 2757-2772.

Hubel, D. H., \& Livingstone, M. S. (1985). Complex-unoriented cells in a subregion of primate area 18. Nature, 315, 325-327.

Hubel, D. H., \& Wiesel, T. N. (1959). Receptive fields of single neurons in the cat's striate cortex. The Journal of Physiology, 148, 574-591.

Hubel, D. H., \& Wiesel, T. N. (1962). Receptive field, binocular interaction and functional architecture in the cat's visual cortex. The Journal of Physiology, 160, 106-154.

Hubel, D. H., \& Wiesel, T. N. (1968). Receptive fields and functional architecture of monkey striate cortex. The Journal of Physiology, 195, 215-243.

Inouye, T. (2000). Visual disturbances following gunshot wounds of the cerebral cortical visual areas: Based on observations of the wounded in the recent Japanese wars, 1904-1905 (M. Glickstein $\&$ M. Fahle, Trans.). Brain, 123(Suppl.). (Original work published 1909)

Kohler, P. J., Caplovitz, G. C., \& Tse, P. U. (2009). The whole moves less than the spin of its parts. Attention, Perception, \& Psychophysics, $71,675-679$.

Krolik, W. (1934). Über Erfahrungswirkungen beim Bewegungssehen. Psychologische Forschung, 20, 47-101.

Lee, B. B., Pokorny, J., Smith, V. C., \& Kremers, J. (1994). Responses to pulses and sinusoids in macaque ganglion cells. Vision Research, 34, 3081-3096.
Lissauer, H. (1889). Ein Fall von Seelenblindheit nebst Beitrage zur theorie derselben. Archiv für Psychiatrie und Nervenkrankheiten, $21,222-270$.

Metzger, W. (1936). Laws of seeing (L. Spillmann, M. Wertheimer, \& S. Lehar, Trans.). Cambridge, MA: MIT Press

Murray, S. O., Boyaci, H., \& Kersten, D. (2006). The representation of perceived angular size in human primary visual cortex. Nature Neuroscience, 9, 429-434.

Roach, N. W., McGraw, P. V., \& Johnston, A. (2011). Visual motion induces a forward prediction of spatial pattern. Current Biology, $21,740-745$.

Schiller, P. H., Finlay, B. L., \& Volman, S. F. (1976a). Quantitative studies of single-cell properties in monkey striate cortex: I. Spatiotemporal organization of receptive fields. Journal of Neurophysiology, 39, 1288-1319.

Schiller, P. H., Finlay, B. L., \& Volman, S. F. (1976b). Quantitative studies of single-cell properties in monkey striate cortex: II. Orientation specificity and ocular dominance. Journal of Neurophysiology, 39, 1320-1333.

Schiller, P. H., Finlay, B. L., \& Volman, S. F. (1976c). Quantitative studies of single-cell properties in monkey striate cortex: III. Spatial frequency. Journal of Neurophysiology, 39, 13341351.

Seriès, P., Georges, S., Lorenceau, J., \& Frégnac, Y. (2002). Orientation dependent modulation of apparent speed: A model based on the dynamics of feedforward and horizontal connectivity in V1 cortex. Vision Research, 42, 2781-2797.

Shapiro, A., Lu, Z.-L., Huang, C.-B., Knight, E., \& Ennis, R. (2010). Transitions between central and peripheral vision create spatial/ temporal distortions: A hypothesis concerning the perceived break of the curveball. PLoS One, 5, e13296.

Shipley, T. F., \& Kellman, P. J. (1994). Spatiotemporal boundary formation. Journal of Experimental Psychology. General, 123, $3-20$.

Shipley, T. F., \& Kellman, P. J. (1997). Spatio-temporal boundary formation: The role of local motion signals in boundary perception. Vision Research, 37, 1281-1293.

Shipp, S., \& Zeki, S. (1985). Segregation of pathways leading from area V2 to areas V4 and V5 of macaque monkey visual cortex. Nature, 325, 322-325.

Tse, P. U. (2006). The neural correlates of transformational apparent motion. NeuroImage, 31, 766-773.

Tse, P. U., \& Caplovitz, G. C. (2006). Contour discontinuities subserve two types of form analysis that underlie motion processing. Progress in Brain Research, 154, 271-292.

Tse, P. U., \& Hsieh, P.-J. (2006). The infinite regress illusion reveals faulty integration of local and global motion signals. Vision Research, 46, 3881-3885.

Tse, P. U., \& Logothetis, N. K. (2002). The duration of 3-D form analysis in transformational apparent motion. Perception \& Psychophysics, 64, 244-265.

Ungerleider, L. G., \& Desimone, R. (1986). Cortical connections of visual area MT in the macaque. The Journal of Comparative Neurology, 248, 190-222.

Ungerleider, L. G., Desimone, R., \& Moran, J. (1986). Asymmetry of central and peripheral field inputs from area V4 to the temporal and parietal lobes of the macaque. Society for Neuroscience Abstract, 12

Ungerleider, L. G., Gattas, R., Sousa, A. P. B., \& Mishkin, M. (1983). Projections of area V2 in the macaque. Society for Neuroscience Abstracts, 152.

Vaina, L. M. (1989). Selective impairment of visual motion interpretation following lesions of the right occipito-parietal area in humans. Biological Cybernetics, 61, 347-359.

Van Essen, J. C., \& Gallant, J. L. (1994). Neural mechanisms of form and motion processing in the primate visual system. Neuron, 13, 1-10. 
Whitney, D. (2002). The influence of visual motion of perceived position. Trends in Cognitive Sciences, 6, 211-216.

Whitney, D., Goltz, H. C., Thomas, C. G., Gati, J. S., Menon, R. S., \& Goodale, M. A. (2003). Flexible retinotopy: Motiondependent position coding in the visual cortex. Science, 302, 878-881.
Zihl, J., von Cramon, D., \& Mai, N. (1983). Selective disturbance of movement vision after bilateral brain damage. Brain, 106, 313-340.

Zihl, J., von Cramon, D., Mai, N., \& Schmid, C. (1991). Disturbance of movement vision after bilateral posterior brain damage: Further evidence and follow up observations. Brain, 114, 2235-2252. doi:10.1093/brain/114.5.2235 